\title{
Accurate, Pre-RTL Temperature-Aware Design Using a Parameterized, Geometric Thermal Model
}

\author{
Wei Huang, Member, IEEE, Karthik Sankaranarayanan, Kevin Skadron, Senior Member, IEEE, \\ Robert J. Ribando, and Mircea R. Stan, Senior Member, IEEE
}

\begin{abstract}
Preventing silicon chips from negative, even disastrous thermal hazards has become increasingly challenging these days; considering thermal effects early in the design cycle is thus required. To achieve this, an accurate yet fast temperature model together with an early-stage, thermally optimized, design flow are needed. In this paper, we present an improved block-based compact thermal model (HotSpot 4.0) that automatically achieves good accuracy even under extreme conditions. The model has been extensively validated with detailed finite-element thermal simulation tools. We also show that properly modeling package components and applying the right boundary conditions are crucial to making full-chip thermal models like HotSpot accurately resemble what happens in the real world. Ignoring or oversimplifying package components can lead to inaccurate temperature estimations and potential thermal hazards that are costly to fix in later designs stages. Such a full-chip and package thermal model can then be incorporated into a thermally optimized design flow where it acts as an efficient communication medium among computer architects, circuit designers and package designers in early microprocessor design stages, to achieve early and accurate design decisions and also faster design convergence. For example, the temperature-leakage interaction can be readily analyzed within such a design flow to predict potential thermal hazards such as thermal runaway. An example SoC design illustrates the importance of adopting such a thermally optimized design flow in early design stages.
\end{abstract}

Index Terms - compact thermal model, early design stages, leakage, parameterized model, temperature, thermally optimized design flow.

\section{INTRODUCTION}

Because of the continued non-ideal scaling of CMOS technology [1], managing on-chip temperatures, especially local hot spots, has become a major challenge. To deal with this thermal challenge, temperature-aware design in early stages, such as microarchitecture design, is especially important, because the architecture definition fixes what subsequent design stages, such as circuit implementation, packaging, etc., must accommodate, and has the greatest impact on final design.

Temperature-aware design in early, pre-RTL (Register Transfer Level) design stages, in turn, requires a fast, yet accurate, architectural thermal model to explore large regions of the design space. Such a thermal model should be "by-construction" and parameterized, i.e., the model is constructed solely based on chip and package geometries and material properties, hence allowing

This work is funded by NSF CRI grant CNS-0551630 and has partial support from a MARCO IFC grant.

W. Huang, K. Sankaranarayanan and K. Skadron are with the Department of Computer Science, University of Virginia.

R. J. Ribando is with the Department of Mechanical and Aerospace Engineering, University of Virginia.

M. R. Stan is with the Charles L. Brown Department of Electrical and Computer Engineering, University of Virginia. a designer to explore potential design choices without the costly, slow building of a prototype [2].

The complicated 3-D heat transfer within both the silicon chip and the package, together with the closely coupled relationship between power (density) and temperature requires that such a thermal model be accurate even under extreme simulated conditions. While better accuracy in general means less computational efficiency, an early-stage, by-construction, fullchip thermal model can still achieve satisfactory accuracy by carefully correcting deficiencies in the model structure that lead to significant errors, without sacrificing the speed advantage from its compact nature. For example, in a microarchitecture floorplan, it is not uncommon to have functional blocks with relatively high aspect ratios. Modeling these high-aspect-ratio functional blocks as single nodes is less accurate than dividing them into a few more sub-blocks with aspect ratios close to unity, as we will see later in this paper. The flexibility in refining a functional block also validates the fact that the intuitive, parameterized and byconstruction modeling paradigm works well.

In addition to modeling the silicon chip, the early-stage compact thermal model should also properly model different package components. Ignoring or over-simplifying package components in a full-chip thermal model can lead to inaccurate temperature estimations and potential thermal hazards that are costly to fix in later design stages. For example, the thermal interface material (TIM) is a thin layer bonding silicon chip and heat spreader. Due to its low thermal conductivity, TIM prevents effective heat spreading from silicon to the rest of the package, and thus exacerbates localized heating within the die. Therefore, ignoring TIM or using the wrong TIM thickness in the model causes unrealistic silicon temperature estimates. Another example is the thermal boundary condition at the heatsink-air interface. Traditional thermal models usually assume an isothermal condition with a single thermal resistor connecting the heatsink surface to ambient air. In reality, a convective boundary condition is more appropriate as the heatsink surface is usually far from isothermal. Using the proper boundary condition can greatly improve the accuracy of the thermal model.

Consequently, an accurate full-chip and package compact thermal model can also act as a convenient medium for enhanced collaborations among circuit, architecture and package designers. This implies a design flow leading to early design evaluations from a thermal point of view. If potential thermal hazards are discovered early in the design process, different design tradeoffs can be carried out at the architecture level, the circuit level and the package level, in an efficient way. For example, it is well-known that subthreshold leakage power is exponentially dependent on operating temperature. An accurate early-stage thermal model can efficiently close the temperature-leakage loop and warn of potential thermal disaster such as thermal runaway very early in the design process. 
In this paper, we address the above topics and make the following contributions:

1) We identify sources of inaccuracies in a by-construction early-stage architecture-level thermal model, and provide solutions to improve the accuracy under extreme conditions such as blocks with high aspect ratios and high power densities. We use the popular HotSpot thermal model [3] as the base case. All the proposed solutions are implemented in the new HotSpot version 4.0 [4].

2) We demonstrate the importance of modeling package components and using a proper thermal boundary condition, leading to a more useful full-chip and package thermal model that accurately resembles the temperature distribution in real processors and other IC designs.

3) We propose a thermally optimized design flow based on HotSpot 4.0 for early design stages. The design flow involves designers at all abstraction levels, who collaborate efficiently with the help of HotSpot, and reach a thermally optimized design with faster design convergence and less design cost. We also show a potential leakage-induced thermal runaway example, which demonstrates the importance of the proposed design flow.

This paper is organized as follows. Section II briefly introduces HotSpot, which is the thermal model we use for experiments and analysis throughout the paper. It also reviews other related work. Section III identifies the weakness of the generic by-construction modeling method, and provides solutions to improve its accuracy. Section IV shows the results of the proposed improvements. Following that, Section $\mathrm{V}$ proposes the thermally optimized design flow that can catch potential thermal hazard such as leakageinduced thermal runaway during early design stages efficiently. Section VI summarizes the work.

\section{RELATED WORK}

The HotSpot [3] thermal model is widely used by the computer architecture research community. To date, HotSpot seems to have been mostly used with existing architectural simulation infrastructures such as SimpleScalar ${ }^{1}$ and Wattch [5], but it is designed as a portable library that can be used with a wide range of modeling infrastructures. HotSpot has a by-construction parameterized structure and is available online ${ }^{2}$.

HotSpot was first introduced only as a block-based model. Later on was also introduced a regular-grid-based HotSpot model [6]. One major reason to develop the grid model was to achieve more accuracy by modeling lateral heat transfer paths in more detail than the block model. The irregular block model of HotSpot is suitable for fast thermal simulations with arbitrarilysized functional blocks. In contrast the HotSpot grid model achieves more detailed temperature estimations at the cost of more computational overhead. The importance of having a grid-like thermal model was also discussed in [7].

There are numerous other existing chip level temperature models besides HotSpot. Among them, the most accurate models are the detailed finite-element models such as $\mathrm{ANSYS}^{3}$, FloWorks ${ }^{4}$ and FreeFEM $3 d^{5}$, which unfortunately are very computationally

\footnotetext{
${ }^{1}$ http://simplescalar.com

${ }^{2}$ http://lava.cs.virginia.edu/HotSpot/

${ }^{3}$ http://www/ansys.com

${ }^{4}$ http://www.solidworks.com/pages/products/cosmos/cosmosfloworks.html

${ }^{5}$ http://www.freefem.org/ff $3 \mathrm{~d} /$
}

intensive and time-consuming. There are also other thermal models dividing silicon into fine meshes and solving with fast solvers, such as [8], [9] and the HotSpot grid model [10]. These models also achieve excellent accuracy whereas still incurring significant computational overhead compared to the parameterized compact thermal models such as the HotSpot block model [2], [3]. On the other hand, the compact thermal models trade off absolute accuracy with simpler structure and speed by constructing the model directly according to functional units of interest and physical properties of the chip. Therefore, they are well suited to fast transient thermal simulations required in computer architecture research. This "by-construction" nature also makes the thermal model parameterized and allows designers to explore hypothetical designs easily without building prototypes.

Regarding transient thermal modeling, another previous work [11] approaches the topic analytically at a finer granularity - the transistor level. Since the size of a transistor is much smaller than the die thickness, silicon can be modeled as semi-infinite, which greatly simplifies the boundary conditions and makes an analytical transient heat transfer solution possible. With the semi-infinite silicon assumption, heat can be fully spread within silicon before reaching the back surface of the silicon substrate, leading to a smaller thermal resistance and also a shorter thermal time constant. On the contrary, the HotSpot model aims at granularities coarser than transistors, and the block size or grid size are usually comparable with or greater than the die thickness, rendering the boundary conditions assumed in [11] not valid. With a finite silicon thickness, the heat generated from a block cannot be fully spread before reaching the back surface of the die, causing a larger thermal resistance and also a longer thermal time constant. This difference in silicon thermal time constant leads to slower transient temperature changes in HotSpot which models larger blocks and grid cells than the model in [11] which models tiny transistors.

So far, models such as the HotSpot block model have been successfully helping computer architects in their temperatureaware research. However, there is still room to improve their accuracy and usefulness further without introducing significant computational overhead. Recently, some accuracy concerns were raised regarding the HotSpot block model [12]. Noticeable and even significant errors were found under certain evaluation scenarios. All of these scenarios contain extreme configurations (e.g. functional blocks with very high aspect ratios) or uncommon in designs (e.g. extremely high power densities). In this paper, we extend the discussions in [4] to analyze the sources of inaccuracies for the by-construction compact thermal modeling approach and provide solutions to improve the accuracy even under the aforementioned extreme conditions, which is an important improvement of our previous work [3], [10], [13].

Another important factor that greatly impacts the accuracy of chip-level thermal models is how accurately the thermal package components are modeled and how realistic the boundary conditions are applied. In recent years, there have been a number of existing full-chip thermal models that provide detailed die temperature distributions, such as [14]-[16]. These models all have detailed temperature distribution information across the silicon die and can be solved efficiently. Unfortunately, a limitation of the above models is that the thermal package is oversimplified. For example, the thermal interface material (TIM) that greatly affects die temperature distribution is not included 
in the models. The bottom surfaces of the silicon substrate, the heat spreader and the heatsink are all treated as isothermal, which significantly deviates from the real world convective thermal boundary condition and introduces errors. On the other hand, properly modeling package components and their boundary conditions can significantly improve the model's accuracy and usefulness. Ignoring or over-simplifying the package components can lead to inaccurate temperature estimations, hence incorrect design decisions. In comparison, there are also several packageonly compact thermal models [17]-[19]. These package models consist of simple networks of thermal resistances, whose values are extracted by data-fitting from the results of accurate but timeconsuming detailed numerical package thermal model simulations (e.g. using the finite element method). Therefore, they are not fully parameterized and cannot be easily used to explore new package designs. In addition, these package thermal models can provide only one or a few die-level temperatures, which is far from enough for fine-grained die-level designs. In this paper, we extend the discussions in [20] to show the importance of modeling both chip and package components in a thermally optimized design flow.

With the improved accuracy and the inclusion of package components, a parameterized compact thermal model can be a convenient communication medium among architects, circuit designers and package designers. In this paper, we also outline a thermally optimized design flow for early design stages. With the proposed design flow, potential thermal hazards such as leakageinduced thermal runaway should be discovered as early in the design process as possible. With the help of a compact chip and package level thermal model, across-die temperature distribution can be estimated at design time, which permits thermally selfconsistent leakage power calculations in an iterative manner as shown in [21], [22]. This is illustrated by an example of potential thermal runaway for a SoC design.

\section{ACCURACY IMPROVEMENTS}

This section identifies weaknesses that have come to light in earlier HotSpot block model when used with extreme simulation parameters such as functional blocks with high aspect ratios, high power densities, etc. We show how to address these issues within the framework of the parameterized, by-construction paradigm. Solutions include further dividing blocks with high aspect ratio into smaller sub-blocks, applying a proper heatsink boundary condition, modeling package components that can cause significant error but have been neglected so far, and others. Experimental results regarding the improvements are shown in Section IV.

\section{A. Aspect Ratio}

First, when a functional block is approximated by only one node in the model, the associated lumped thermal resistors and capacitors cannot fully model the distributed nature of heat transfer. In particular, for blocks with high aspect ratios where the lateral heat transfer in one direction dominates the other direction, the resultant error can be more significant. This simply requires higher spatial resolution, and the solution is to further divide these high-aspect-ratio blocks into sub-blocks with aspect ratios closer to unity. In Fig. 1(a), a functional block with high lateral aspect ratio is represented by only one node. The four lumped lateral thermal resistors connected to that node are also shown.
In Fig. 1(b), this block is divided into several sub-blocks with close-to-unity aspect ratios. With this modification, the lateral heat transfer within the block is modeled by a finer network with greater fidelity.

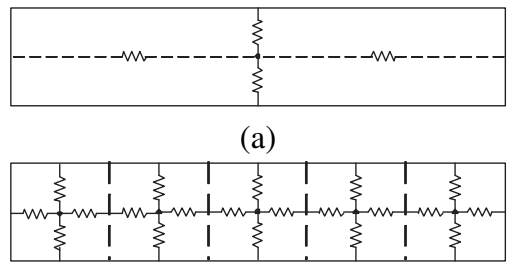

(b)

Fig. 1. A block with high aspect ratio-(a) Only one node represents the block for computational efficiency. (b) The block is divided into sub-blocks with aspect ratio close to unity. The lateral heat transfer paths are modeled with more detail but also more computational complexity.

\section{B. Heatsink Boundary Condition}

Different boundary condition assumptions lead to different temperature estimations. For example, at the heatsink-ambient interface, an isotherm condition is usually assumed in traditional thermal model approaches, whereas a more realistic boundary condition is a convective one, which leads to non-isotherm temperature distribution at the heatsink surface. Therefore, this more realistic convective boundary condition should be adopted to further improve accuracy.

Fig 2(a) shows the model structure in traditional thermal models such as HotSpot 3.1, in which the center part of the upper surface of the heat spreader is approximated to be isothermal and has only one node (each black dot is a node). The heatsinkambient interface also has only one node. In the real case, these surfaces are not fully isothermal. Accuracy can therefore be improved by removing the isothermal nodes and modeling the heatsink at the same level of details as the silicon die. Furthermore, the convection interface between heatsink and ambient air can be modeled with multiple convection surfaces (hence, multiple nodes) with a constant heat transfer coefficient.

$$
R_{\text {convec } i}=\frac{1}{h A_{i}}
$$

where $R_{\text {convec } i}$ is the convection thermal resistance for the $i$ th sub-area of the heatsink convection surface, $h$ is the constant heat transfer coefficient, and $A_{i}$ is the sub-area. The resulting thermal model structure is shown in Fig. 2(b). The heat transfer coefficient $h$ in (1) can be found by solving $h$ from $R_{t o t}=1 /\left(h A_{t o t}\right)$ to make sure the equivalent total convection thermal resistance calculated using the total heatsink surface area $\left(A_{t o t}\right)$ is the same as the lumped isothermal sink-to-air thermal resistance $\left(R_{t o t}\right)$, which is usually specified in a heatsink's datasheet. Modeling the heatsink with more details introduces more computing overhead to the model. However, as long as the floorplan does not contain too many blocks, the overhead remains tolerable.

Similarly, a recent full-chip thermal model [23] also has added more nodes in the package components. The authors of [23] approximate the convective boundary condition by mapping and splitting heat spreader and heat sink into blocks according to the die floorplan, with each block in the package bigger than its silicon counterpart as a result of the bigger size of spreader and sink than the silicon die. This is a natural way to add 


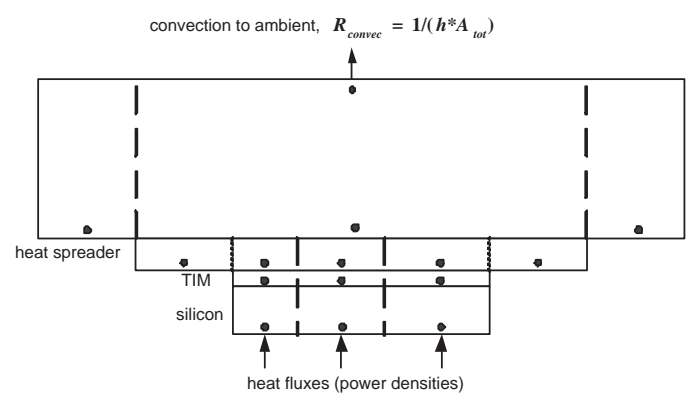

(a)

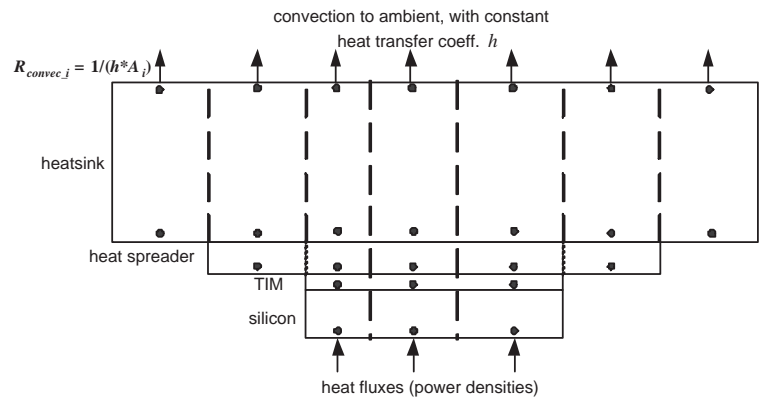

(b)

Fig. 2. (a) Simple thermal model with only one convection resistor from heatsink to ambient air, with top surface of heat spreader and heatsink both assumed to be isothermal. (b) An improved model structure. The center part of heatsink is modeled at the same level of detail as the silicon. The isotherm nodes are replaced with multiple nodes connected by different convection resistors.

more details in the package components and achieves reasonable accuracy. This package components splitting scheme is slightly different from HotSpot-HotSpot only divides the center parts of spreader and sink covered by the previous layer into the same number of blocks as the previous layer, and use four extra nodes for the remaining peripheral areas. The reason behind this is the fact that finite-element simulations (e.g. ANSYS) show that, for copper spreader and heat sink, since the heat spreading within copper is significantly better than silicon, the temperatures outside the center parts of the spreader and sink quickly drop to uniform values. Therefore, we find it is more accurate to split the package into center nodes and peripheral nodes. For other types of spreaders and heat sinks, such as those with different thermal conductivities, phase-change spreaders and micro-channel spreaders and sinks, different schemes of modeling the package components may need to be developed on a case-to-case basis.

\section{Including Thermal Interface Material (TIM)}

As mentioned in Section II, ignoring or over-simplifying package components can introduce significant errors to the results of a thermal model. One package component, the thermal interface material (TIM), is of particular interest. TIM is special because it has rather low thermal conductivity due to material limitation and economic reasons. Comparing with the thermal conductivity of silicon (about $100 \mathrm{~W} / \mathrm{m}-\mathrm{K}$ ), typical TIM thermal conductivity is less than $10 \mathrm{~W} / \mathrm{m}-\mathrm{K}$ nowadays [24]. In addition, TIM is the layer usually between silicon die and the heat spreader. Therefore, a low-conductivity TIM prevents efficient heat spreading within the silicon and exacerbates the on-chip local hot spot temperatures. Although TIM with better thermal conductivity is being developed, it will remain as a concern at least for the near future.

\section{Additional Improvements to HotSpot}

Specific to HotSpot, the following additional sources of accuracy we identified and solutions are proposed here.

First, transient thermal responses can be inaccurate when high power density is applied to a block. In general, absolute transient accuracy is harder to achieve than static accuracy in HotSpot without introducing significant extra model complexity. This is due to the lumped structure of HotSpot and the distributed nature of actual transient thermal response. In HotSpot, scaling factors to thermal capacitors are used to match the thermal time constants between lumped and distributed systems. However the scaling factors cannot guarantee perfect match over the entire transient temperature response. The only way to achieve ultimate transient accuracy is to use a very fine 3-D mesh to model the system, which inevitably introduces significant computational overhead, and is probably not suitable for architecture-level simulations. Here, we improve the transient accuracy of HotSpot by using a constant 0.5 scaling factor for lumped thermal capacitors. As will be shown in Section IV-A.3, using a constant 0.5 capacitance scaling factor in the model achieves fairly good accuracy with respect to ANSYS for most of the time scales. The reason behind the 0.5 scaling factor is that the time constant of a distributed resistor-capacitor circuit is half of that of a one-lumped resistorcapacitor stage [25].

Another source of inaccuracy in HotSpot comes from the fact that certain material properties, such as thermal conductivity and specific heat, are weakly temperature dependent. Approximating them with constant values thus introduces small errors. Although it is fairly straightforward to include this in HotSpot in the form of lookup tables, this is not the focus of this paper and is a topic for future work.

To accurately take the temperature-leakage dependency into consideration during early design stage, HotSpot 4.0 is further extended to calculate the leakage power according to updated temperature, using user's own leakage model or HotLeakage [26] and checking for convergence or thermal runaway.

\section{RESUlTS OF ACCURACY IMPROVEMENTS}

In this section, we present the experimental results of the effect of the above-mentioned solutions to the accuracy concerns regarding a by-construction parameterized compact thermal model such as HotSpot. All the improvements are implemented and verified in HotSpot version 4.0. For better clarity, we isolate the results of TIM's impact on temperature estimates to Section IV$\mathrm{B}$, while showing results of all the rest solutions combined in Section IV-A.

\section{A. Chip and Boundary Condition Solutions}

To evaluate the accuracy improvement, we use ANSYS as our primary reference finite-element model and FreeFEM3d as a secondary source. ANSYS allows users to better control on the level of spatial discretization (mesh granularity) and the shape of the finite element (e.g. tetrahedral vs. quadrilateral elements) so that greater accuracy can be achieved with smaller elements. In our ANSYS experiments, we use multiple meshing levels (e.g. 15 layers for silicon) and types of elements (e.g. tetrahedral vs. quadrilateral elements with up to 20 nodes per element), and ensure that the results are consistent across them. The results of FreeFEM3d are either from repeating experiments in [12] or extracted directly from [12]. 
1) ALPHA EV6 Steady-State Results: The package geometry used is similar to Fig. 2. For this experiment, the silicon die has $16 \mathrm{~mm} \times 16 \mathrm{~mm} \times 0.5 \mathrm{~mm}$ dimensions. The thermal interface material (TIM) layer has the same size as the die and is $0.1 \mathrm{~mm}$ thick. We also use two different TIM materials, one has a better conductivity of $7.5 \mathrm{~W} / \mathrm{m}-\mathrm{K}$ (good TIM); the other has a worse thermal conductivity of $1.33 \mathrm{~W} / \mathrm{m}-\mathrm{K}$ (worse TIM).

The heat transfer coefficient at the top surface is $2777.7 \mathrm{~W} / \mathrm{m}^{2}$ $\mathrm{K}$, which is equivalent to a single lumped convection thermal resistance of $0.1 \mathrm{~K} / \mathrm{W}$. The floorplan is one that is similar to that of EV6. We slightly modify the coordinates of the functional blocks for alignment so that it is easier to build the model in ANSYS and FreeFEM3d. We use the same modified EV6 floorplan for HotSpot, ANSYS and FreeFEM3d in this experiment. The floorplan is shown in Fig. 3.

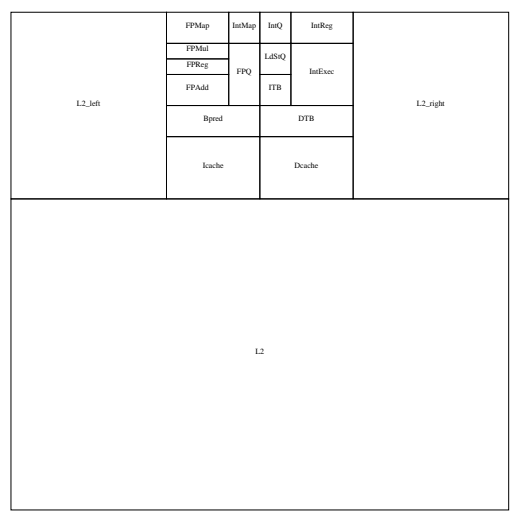

Fig. 3. EV6 floorplan, adapted from [3].

Fig. 4(a) and Fig. 5(a) show the temperature estimations from ANSYS, FreeFEM3d (FF3d), HotSpot3.1 $1^{6}$ and HotSpot 4.0 for the good TIM and the worse TIM. To better illustrate the absolute errors of HotSpot block model, in Fig. 4(b) and Fig. 5(b), we use ANSYS temperatures as the references and plot the errors of HotSpot4.0, HotSpot3.1 and FreeFEM3d (FF3d) with respect to ANSYS for both TIM materials.

There are several observations from Fig. 4 and Fig. 5:

1) HotSpot 4.0 in general has lower error than HotSpot 3.1. The improved accuracy is achieved by eliminating the isotherm nodes in package and dividing high-aspect-ratio blocks into sub-blocks with unit aspect ratios.

2) For the case of good TIM, HotSpot is even closer to ANSYS than FreeFEM3d! Furthermore, even HotSpot 3.1 does provide reasonably accurate temperature estimations. Since the package configuration with good TIM represents a realistic package for modern high-performance microprocessors, we can see that the original HotSpot 3.1 block model is already quite accurate under typical thermal simulation scenarios.

3) For the case of worse TIM, HotSpot predicts hotter temperatures than both ANSYS and FreeFEM3d in most cases, but the percentage errors for hot units, e.g. BPred and IntReg, are $3.05 \%$ and $2.56 \%$, respectively. Overall worstcase percentage error with worse TIM is $11.96 \%$ for ICache, which is a relatively cool unit.

4) There are noticeable differences between ANSYS and

\footnotetext{
${ }^{6} \mathrm{HotSpot} 3.1$ is an earlier version which has TIM but does not include the other proposed solutions.
}

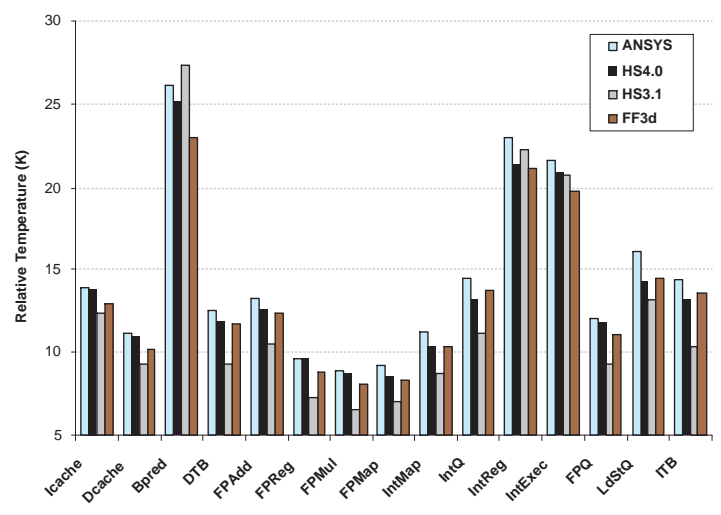

(a)

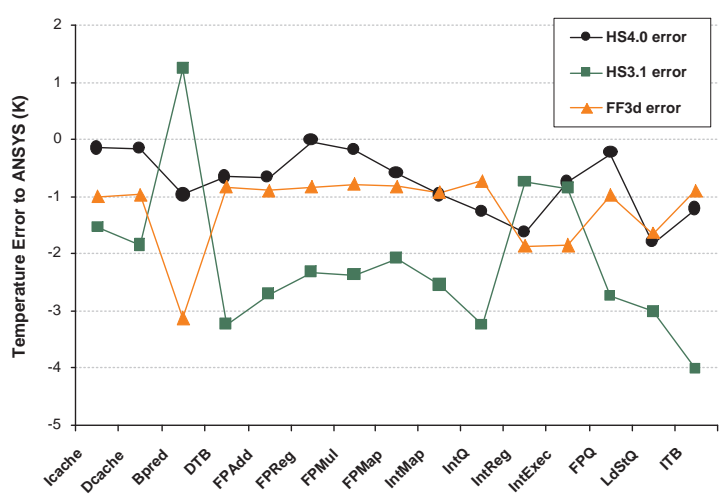

(b)

Fig. 4. (a) EV6 block relative temperatures with good thermal interface material.(b) EV6 block relative temperature errors with respect to ANSYS, with good thermal interface material $\left(k_{T I M}=7.5 \mathrm{~W} /(\mathrm{m} \cdot \mathrm{K})\right)$.

FreeFEM3d (FF3d) as well, both being detailed finiteelement models.

2) Square Source Steady-State Results: A better experiment that helps to evaluate and explain the steady-state errors is to test a range of heat source sizes with the same power density. In this experiment, the silicon chip has a size of $21 \mathrm{~mm} \times 21 \mathrm{~mm} \times 0.5 \mathrm{~mm}$, and the dimensions of other package components are the same as Section IV-A.1. The center heat source size varies from $1 \mathrm{~mm}$ to $19 \mathrm{~mm}$. The applied power density to the center block is set to a constant value of $1.66 \mathrm{~W} / \mathrm{mm}^{2}$. Fig. 6(a) shows a floorplan with a $1 \mathrm{~mm}$ square heat source together with its high aspect ratio neighbor blocks. Fig. 6(b) shows the same floorplan in which the high aspect ratio blocks are divided into square sub-blocks.

Fig. 7 and Fig. 8 show the comparisons among the HotSpot 3.1, HotSpot 4.0, ANSYS and FreeFEM3d for different heat source sizes. We also plot the HotSpot 3.1 results with unity-aspect-ratio (sub)blocks (HS3.1-AR) to isolate the effect of each individual aforementioned modifications (i.e. unity aspect ratio and nonisothermal boundary condition). As can be seen, the HotSpot 4.0 block model is much more accurate than the earlier HotSpot 3.1 block model.

For smaller heat source size $(1 \mathrm{~mm}$ to $5 \mathrm{~mm})$, the significant error of HotSpot 3.1 is caused by the extreme aspect ratio (10:1) of the four long and narrow blocks that are adjacent to the center small heat source block. In HotSpot 4.0, these long, narrow blocks 


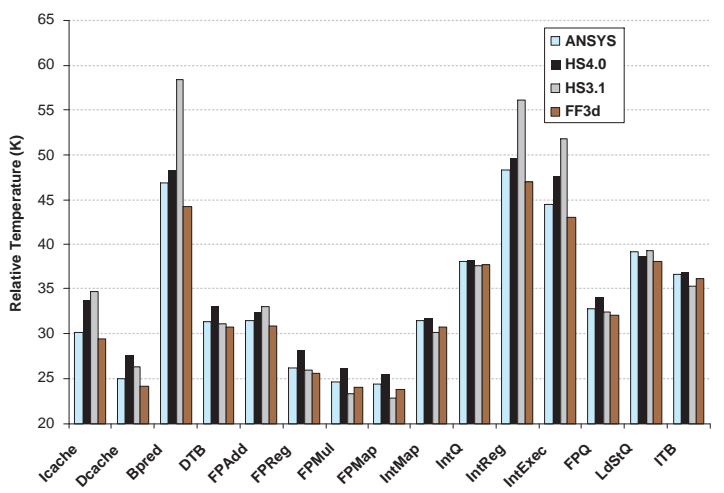

(a)

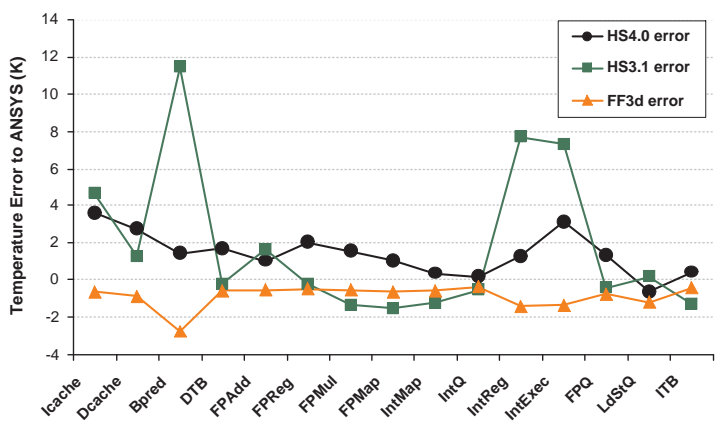

(b)

Fig. 5. (a) EV6 block relative temperatures with worse thermal interface material.(b) EV6 block relative temperature errors with respect to ANSYS, with worse thermal interface material $\left(k_{T I M}=1.33 \mathrm{~W} /(m \cdot K)\right)$.

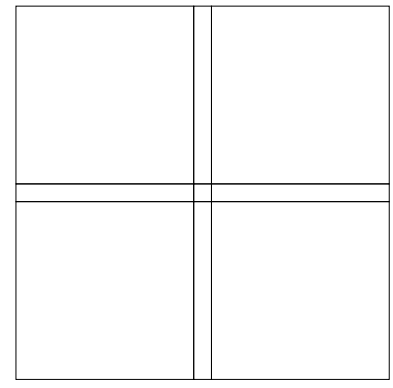

(a)

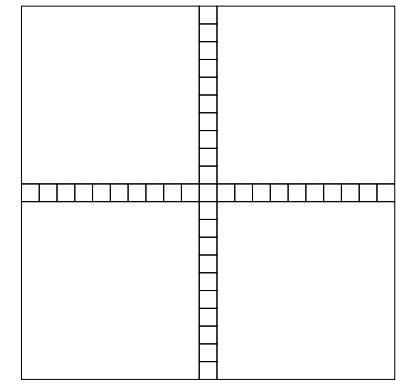

(b)
Fig. 6. (a) Floorplan with $1 \mathrm{~mm}$ center square heat source dissipating $1.66 \mathrm{~W}$. Notice the neighboring high aspect ratio blocks. (b) The neighboring high aspect ratio blocks are divided into square sub-blocks.

are automatically divided into 10 sub-blocks with aspect ratios of $1: 1$, thus the accuracy is greatly improved (see left part of the "HS3.1 AR" curves for small heat source sizes).

For larger heat source size (e.g., 19mm, which has $600 \mathrm{~W}$ of power!), the significant error of HotSpot 3.1 is caused by the fact that the upper surfaces of the heat spreader and the heatsink are no longer close to being isothermal, so approximating them with single nodes yields significant errors. In HotSpot 4.0, the isothermal nodes are removed. Instead, we model the heatsink at the same level of detail as the silicon die and use a constant heat transfer coefficient $\left(\mathrm{h}=2777.7 \mathrm{~W} / \mathrm{m}^{2}-\mathrm{K}\right)$ for each sub-area of the heatsink-ambient interface. This significantly improves the accu- racy for large-size heat sources (see the significant improvement for larger heat source sizes from "HS3.1 AR" to "HS4.0").

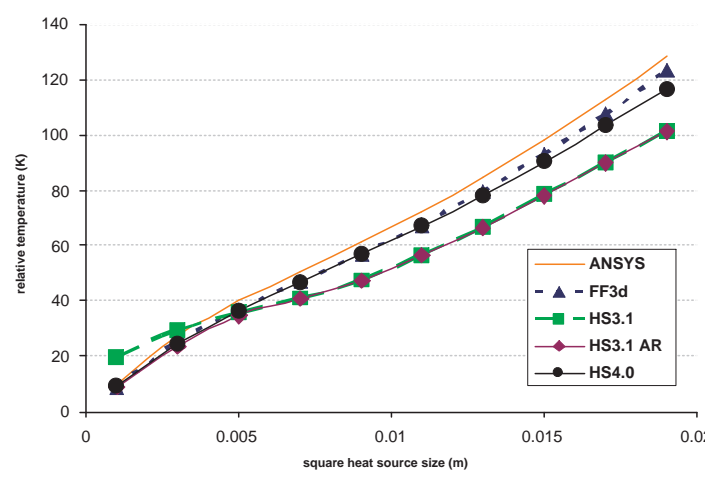

Fig. 7. Center temperature for different heat source sizes, with good thermal interface material $\left(k_{T I M}=7.5 \mathrm{~W} /(\mathrm{m} \cdot \mathrm{K})\right)$, power density is $1.66 \mathrm{~W} / \mathrm{mm}^{2}$.

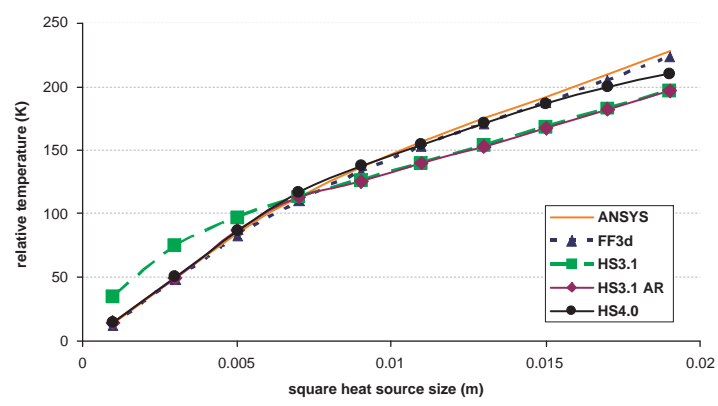

Fig. 8. Center temperature for different heat source sizes, with worse thermal interface material $\left(k_{T I M}=1.33 \mathrm{~W} /(\mathrm{m} \cdot \mathrm{K})\right)$, power density is $1.66 \mathrm{~W} / \mathrm{mm}^{2}$.

Here, again, by eliminating the isothermal nodes in package and dividing high-aspect-ratio blocks into sub-blocks with unit aspect ratios, the HotSpot block model greatly improves its accuracy.

3) Pulse Response for Bpred Unit in EV6 Floorplan: To evaluate the transient accuracy improvement of HotSpot 4.0, we performed an experiment with power pulses of different time scales.

In Fig. 9, power pulses of $100 \mu \mathrm{s}, 1 \mathrm{~ms}$ and $10 \mathrm{~ms}$ are sequentially applied to the Branch Predictor (Bpred) block in the EV6 floorplan with uniform power density of $2 \mathrm{~W} / \mathrm{mm}^{2}$ to verify HotSpot 4.0's accuracy at different time scales. Notice the time axis is in log scale. We compare HotSpot 4.0 and HotSpot 3.1 results with ANSYS. As can be seen, HotSpot 4.0 significantly improves transient accuracy for all time scales under this highaspect-ratio and high-power-density extreme case.

We can see that in addition to eliminating the isothermal nodes in package and dividing high-aspect-ratio blocks into sub-blocks with unit aspect ratios, HotSpot block model's transient accuracy is also improved by using a constant scaling factor of 0.5 to approximate the thermal time constant of the distributive nature of transient temperature evolvement. The scaling factor comes from the analogous electrical distributed RC circuit whose time constant is half of the one-ladder RC circuit [25].

Based on the above steady-state and transient experiments and comparisons among HotSpot block model, ANSYS and 


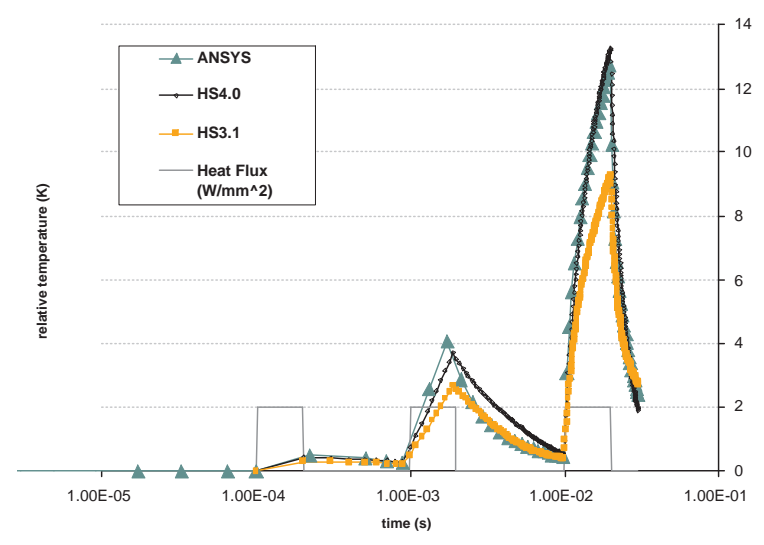

Fig. 9. Transient temperature response for different power pulse widths applied to the branch predictor of EV6. Power density is $2 \mathrm{~W} / \mathrm{mm}^{2}\left(k_{T I M}=\right.$ $7.5 \mathrm{~W} /(m \cdot K))$.

FreeFEM3d, we can see that the improved HotSpot 4.0 model, is accurate as a by-construction compact thermal model for architecture-level and other early-stage design levels. The small inaccuracies come from the fact that the compact thermal model trades off accuracy to achieve greater model compactness.

\section{B. TIM's Impact on Chip Temperature}

Earlier in the paper, we have mentioned that package components can greatly affect the temperature distribution across the silicon die. In this section, we show some example thermal analysis regarding one particular packaging component-thermal interface material (TIM) that bonds the silicon die to the heat spreader.

With the flexibility of the improved parameterized compact thermal model, we can easily investigate the thermal impacts of different TIM properties, such as its thickness, void size, and attaching surface roughness, in early design stages and provide important insights for computer architects, circuit designers and package designers.

We first show how the thickness of TIM affects silicon die temperature distribution. Fig. 10 plots the across-die temperature difference from the compact thermal model with different TIM thickness.

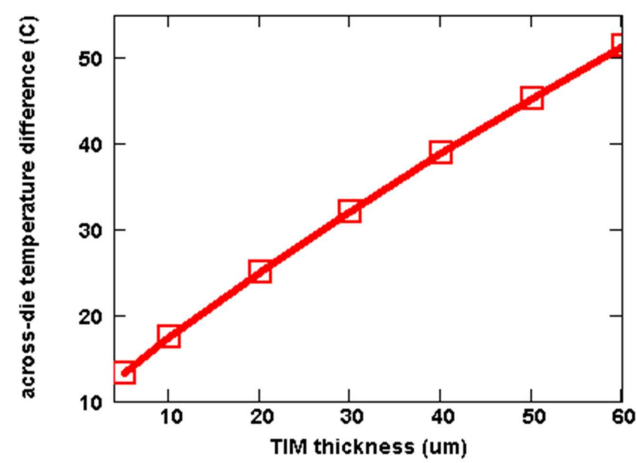

Fig. 10. The impact of thermal interface material (TIM) thickness to silicon die temperature difference [20].

As can be observed from Fig. 10, thicker TIM results in poor heat spreading which leads to large temperature differences across the die. We can see that thick TIM can lead to very large die temperature difference across the die $\left(>50^{\circ} \mathrm{C}\right)$. Even with nominal TIM thickness, which is $20 \mu \mathrm{m}$ for this design, the temperature difference across the die is still $24^{\circ} \mathrm{C}$. This means that the bottom surface of the die can not be modeled as an isothermal surface. If the TIM is thick enough, the resultant extremely large temperature differences across the die may be disastrous to circuit performance and die/package reliability. Using a better heatsink will only lower the average silicon temperature but will not help to reduce the temperature difference. This analysis suggests that using the thinnest possible TIM is one of the key issues for package designers to consider. On the other hand, with the known TIM thickness that can be best assembled in package with state-of-the-art packaging technology, it is the task of circuit designers and computer architects to design proper circuits and architectures to maintain the temperature difference across die within a manageable level.

As another example, Fig. 11 shows the relationship between the size of TIM void and the hot spot temperature. During the packaging process, it is almost unavoidable to leave voids or air bubbles in the thermal interface material. In the compact thermal model, the void in TIM can be easily modeled by introducing higher vertical TIM thermal resistance to the grid cell where the void resides. Different sizes of the TIM void can be modeled by different sizes of the grid cell. For the simulations of Fig. 11, we put the TIM void right under the hottest grid cell, thus modeling the highest possible die temperature in the presence of a void with different sizes. As can be seen from Fig. 11, if the hot spot temperature of the design is $95^{\circ} \mathrm{C}$, a void or air bubble in the TIM with a size of $0.25 \mathrm{~mm}^{2}$ can make the hot spot temperature drastically higher $\left(290^{\circ} \mathrm{C}\right)$, which inevitably leads to thermal runaway of the chip. Therefore, it is desirable to improve the packaging techniques to make the size of the TIM void as small as possible. Package designers usually have the expertise to know typical TIM void sizes for different packaging processes. They can include this information in the thermal model. By doing this, the thermal model is now able to provide possible worstcase temperature regarding TIM void defects. The consequent architecture and circuit design decisions can thus avoid potential thermal hazards caused by the TIM void defects.

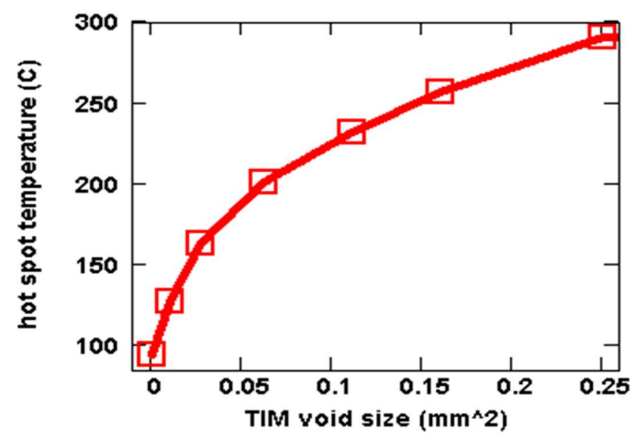

Fig. 11. The impact of the size of void defect in thermal interface material (TIM) to silicon die hottest temperature. Temperatures are normalized to the ideal case where there is no void defect in the TIM layer. TIM void sizes are with the unit of $\mathrm{mm}^{2}[20]$.

Another important thermal interface material property that affects the die temperature is the surface roughness, i.e. nonuniform TIM. In real-life chip packaging process, the bottom surface of the die and the TIM's attaching surface cannot be 
perfectly smooth. As shown in Fig 12, TIM is only attached to the die at the bumps of the TIM surface. This causes ineffective heat conduction and hence higher die temperature comparing to the case where TIM and the die attach to each other perfectly. In order to investigate the impact of TIM non-uniformity to the die temperature, we change the thermal model of the TIM layer according to Fig 12, where we simply model the non-uniformity of the TIM surface as tiny bumps with spacing $2 L$. The size of each grid cell is set to $L$. Therefore, heat can only be conducted through the grid cells representing the touching bumps. Grid cells representing the valleys are essentially tiny voids that do not touch the die and have extremely low thermal conductivity. The value of $L$ thus can be used as an indicator of the non-uniformity of the TIM surface-the surface is rougher when $L$ is larger and vice versa. Fig. 13 is the model results showing the relationship between $L$ (non-uniformity) and die temperatures, where $L=0$ means the TIM surface is perfectly uniform. As observed, even tiny non-uniform TIM surface (e.g. $\mathrm{L}=5 \mu \mathrm{m}$ ) can significantly raise both the hottest and the average die temperature (by about 10 degrees). Package designers again usually have the specifications of the surface non-uniformities for different packaging processes. Without considering such package processing specifications, it is inevitable that a thermal model underestimates the die temperature and leads to designs that are not thermally optimized and designs with higher probability of premature failures.

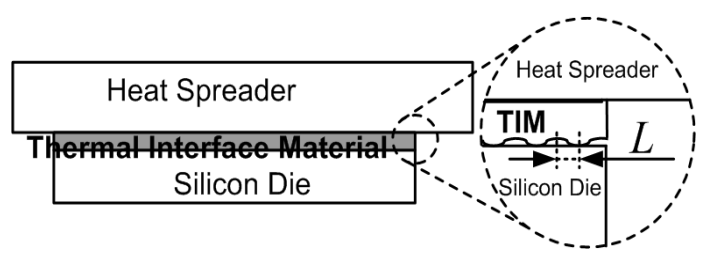

Fig. 12. Close-up view of the TIM/die attaching surface. Surface nonuniformity is indicated by $L[20]$.

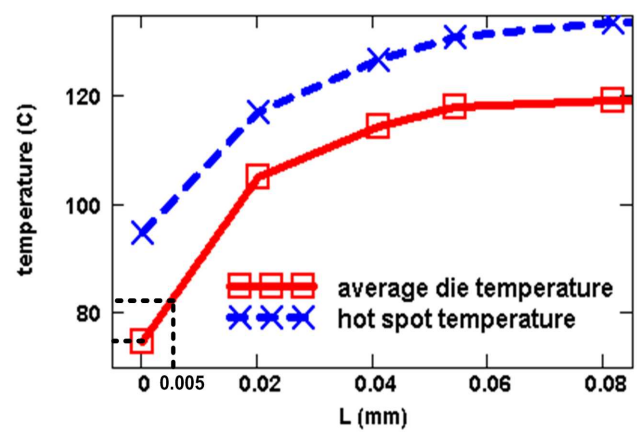

Fig. 13. Hottest die temperature and average die temperature vs. the nonuniformity of TIM attaching surface. The larger $L$ is, the rougher the attaching surface. $L$ is defined in Fig. 12 [20].

\section{Tradeoffs of Using HotSpot 4.0}

While HotSpot 4.0 has better accuracy under more extreme conditions, it also introduces more computational overhead than HotSpot 3.1 due to the increased number of nodes in both silicon and package components. The overhead is usually negligible when the number of blocks is relatively small. HotSpot 4.0 is extremely useful when there are very high-power density blocks with high aspect ratio (e.g. BPred and IntReg with lowconductivity TIM in Fig. 5) or the total power of the chip is extremely high (e.g. the right-end points in Fig. 7 and Fig. 8 corresponding to the $19 \mathrm{~mm} \times 19 \mathrm{~mm} 600 \mathrm{~W}$ heat source). However, if the floorplan has tens and hundreds of blocks, HotSpot 3.1 may be a better tradeoff between computation complexity and accuracy.

\section{A Thermally Optimized Design Flow}

As temperature management is more challenging as the result of the non-ideal CMOS scaling, considering thermal issues early in the design process becomes imperative. Even though the recent trend toward many-core chips to some extent can alleviate localized heating due to a more uniform power distribution compared to traditional single- and dual-core designs, accurately modeling local temperature variation using HotSpot is still important due to the fact the high-activity cores are usually surrounded by cool local caches, hence local temperature distribution may still be far from uniform. In addition, wafer thickness also scales down, resulting in less efficient within-silicon heat spreading and possibly more prominent localized heating, not to mention multicore chips with heterogeneous cores that can vary significantly in terms of power consumption and temperature among different cores.

The HotSpot model is unique because it efficiently models both chip and package temperatures with satisfactory accuracy for any type of processor designs, at any level of details. This is the key to more effective collaborations among computer architects, circuit designers, and package designers. With the help of such an accurate full-chip and package compact thermal model, an earlystage thermally optimized design flow is proposed in this section to accurately predict potential thermal hazards and to achieve economical designs with faster design convergence.

\section{A. The Design Flow}

Fig. 14 illustrates the proposed pre-layout design flow. As shown in Fig. 14, circuit designers first design basic blocks, such as macros, and each macro has a simulated dynamic power for a certain workload. It also has an estimated layout bounding box. Computer architects then assemble a preliminary microarchitecture-level floorplan. At this point, initial total power, including rough estimation of leakage power, can be used for a package designer to propose a preliminary package design. All the information about power, floorplan and package are used to construct a compact thermal model which can perform thermally selfconsistent leakage power calculations as shown in the high-lighted inner loop of Fig. 14. The resulting temperature map can then be utilized to perform temperature-critical reliability analysis (e.g. interconnect electromigration, gate-oxide breakdown and package deformation) and temperature-related performance analysis (e.g. interconnect and device delay, power grid $I R$ drop).

The results of all this analysis, together with the total power, are then compared to the design goals. If the goals are not satisfied, different tradeoffs can be made-circuit designers may need to invent novel circuits with lower power dissipation, computer architects may have to think more about new architectures and different floorplans to better manage power and temperature, or package designers may need to propose more advanced, usually more expensive, packages. On the other hand, if the design goals 


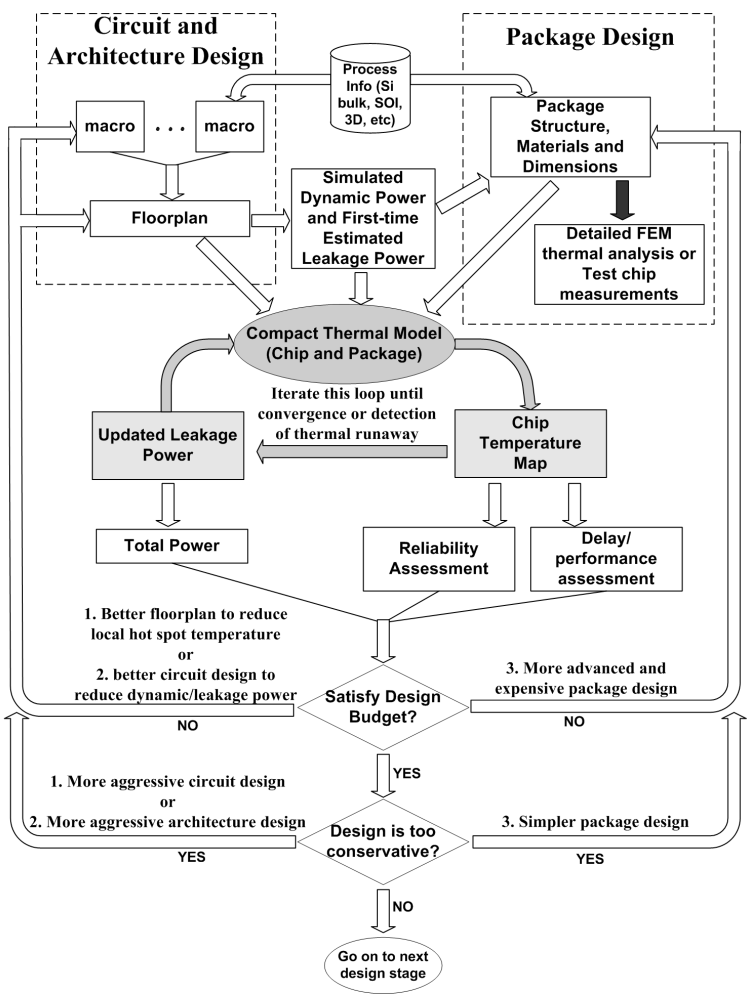

Fig. 14. A design flow showing the compact thermal model acts as a convenient medium for productive collaborations for designers at the circuit, architecture and package levels [20].

are fully satisfied, we still need to check whether the design is too conservative and the design margin is too large for the application. We can then improve the conservative design by either introducing more aggressive circuit and/or architecture solutions to enhance performance, or using simpler and cheaper packages to reduce the cost of the final product. These decisions and tradeoffs can then be evaluated using the thermal analysis again following the same flow until an optimal design point is reached. Then one can proceed to the physical design stage.

With the above design flow, the potential thermal hazards can be discovered and dealt with early and efficiently, thus the design is optimized from a thermal point of view.

\section{B. A SoC Design Example}

To illustrate the importance of adopting such a thermally optimized design flow early in the design process, we show the thermal analysis together with the temperature-leakage loop for a SoC design. We use InCyte ${ }^{\circledR}$, a novel commercialized early design estimation tool ${ }^{7}$ to reconstruct a SoC design based on the published 180nm design data in [27]. This SoC design does not have an integrated heatsink due to its low power consumption. It uses natural convection from a metal covering lid, which acts as the heat spreader, as the cooling method.

We use HotSpot 4.0 for the thermally self-consistent leakage analysis of this SoC design. Because a heatsink is not present in the package, we apply the natural convection boundary condition at the surface of the thin lid that is attached to the silicon substrate. Notice that without the improvement of directly modeling the

${ }^{7}$ http://www.chipestimate.com/ convection boundary condition in HotSpot 4.0, it is impossible to accurately simulate such a scenario because under natural convection, the package surface is obviously not isothermal.

We pick logic and memory modules similar to those in [27] from InCyte's incorporated IP libraries and come up with an early SoC design whose total power is almost identical to data reported in [27]. InCyte also outputs a preliminary floorplan for the design. Although the area of each block is also similar to the original design, the relative locations of different blocks are noticeably different. This is acceptable since InCyte is a tool for early-stage design. Also notice that InCyte estimates leakage power of each block at a constant temperature. Following that, we use HotSpot to estimate chip temperature distribution and pick a proper package from InCyte's package library for this design based on data estimated from InCyte. If we assume the on-chip highest temperature constraint is $85^{\circ} \mathrm{C}$ and the ambient temperature $25^{\circ} \mathrm{C}$, we find that the thermal package needs to have a lumped thermal resistance of $18.2 \mathrm{~K} / \mathrm{W}$, which is common for standard SBGA packages, in order to keep the hot spot temperature below $85^{\circ} \mathrm{C}$. The estimated temperature map of this $180 \mathrm{~nm}$ design is shown in Fig. 15.

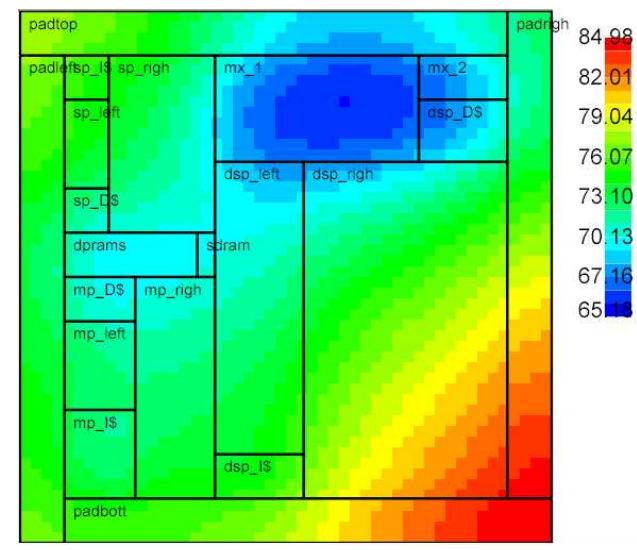

Fig. 15. Estimated temperature map of an SoC design at $180 \mathrm{~nm}$ technology, based on data in [27] and InCyte. Temperatures are in ${ }^{\circ} \mathrm{C}$.

Because InCyte does not yet include the temperature dependency of leakage, whereas sub-threshold leakage is exponentially dependent on temperature, we double-check to see whether the thermally self-consistent leakage power causes thermal problems to this $180 \mathrm{~nm}$ SoC design. Using HotSpot and the simplistic leakage model in [20] to iterate the temperature-leakage loop as shown in Fig. 14, after convergence we find that the final total leakage is only a negligible $546 \mu \mathrm{W}$ for this design with the picked package. Therefore, the above temperature estimation is quite accurate without considering the temperature-leakage loop.

However, if we re-design this SoC design in 90nm technology, there are two design possibilities: 1) We scale both the area and active power of each individual blocks and thus maintain the same function and complexity. This means that the total power of the entire design is also scaled accordingly, thus the power density remains the same due to area scaling. Therefore, we can use a cheaper thermal package for less overall power consumption and keep the chip below the 85C thermal constraint. 2) Since ITRS [1] projects that the die size and power remain the same, if not increasing, across different technology nodes, we can alternatively assume the total active power and chip area remain the same 
as those in the $180 \mathrm{~nm}$ design. Assuming a floorplan similar to that in $180 \mathrm{~nm}$ technology, this is equivalent to adding more parallelism (such as more processing cores and higher memory bandwidth) to the die and designing the chip for higher throughput by burning more power. In this case, with the same $18.2 \mathrm{~K} / \mathrm{W}$ thermal package, after iterating the leakage-temperature loop, the hottest on-chip temperature exceeds the thermal threshold and eventually causes thermal runaway! The reason is two-fold: 1) at $90 \mathrm{~nm}$, a greater fraction of total power consumption is caused by leakage [1], and 2) the subthreshold leakage power's dependency on temperature is stronger at $90 \mathrm{~nm}$ than at $180 \mathrm{~nm}$ (see the leakage model coefficients shown in [28] and [29]). The results are listed in Table I.

The above SoC design example shows that it is crucial to incorporate thermal estimations (such as leakage-temperature dependence) early in the design process in order to locate potential thermal hazards that are too costly to fix in later design stages. At this early design stage, possible solutions to the SoC design at $90 \mathrm{~nm}$ can be: 1) circuit designers can choose IPs that have highVt transistors, and use reverse body-bias or sleep transistors for non-critical paths to reduce leakage. 2) architects can consider using dynamic voltage and frequency scaling (DVFS), migrating computation [3] [28], more parallelism, and temperatureaware floorplanning techniques [30], etc., to reduce hot spot temperatures. Alternatively, 3) package designers need to consider the possibility of adding a heatsink or a fan. Tradeoffs among portability, cost, performance and temperature have to be made in this case by following the design flow in Fig. 14. Unlike HotSpot 4.0, other existing thermal modeling approaches are either not accurate enough (e.g. neglecting package component details or using the wrong boundary conditions) or too timeconsuming (e.g. detailed FEM), hence are not suitable for such design tradeoff analysis early in the design process.

\section{CONCLUSIONS}

In this paper, we first present improvements to an efficient byconstruction compact thermal model, like HotSpot, to make it accurate even under scenarios such as high aspect ratio blocks, high power density, and to better model realistic convective boundary conditions for thermal package components. The accuracy improvements of both steady-state and transient temperatures are confirmed by comparing with finite-element models in ANSYS and FreeFEM3d. The importance of accurately considerations and modeling of package components also determines the accuracy of the die-level temperature estimations. Several examples are presented to illustrate the impact of thermal interface material (TIM) on die temperature distribution. With the improvements of the model structure and the proper inclusion of package components, thermal models such as HotSpot 4.0 can further act as a convenient communication medium for more efficient cooperations among computer architect, circuit designers and package designers, thus achieving a thermally optimized design early in the design stages. The importance of adopting such an early-stage thermally optimized design flow is illustrated by the detection of potential thermal runaway in the early-stage analysis for a $90 \mathrm{~nm}$ SoC design.

\section{ACKNOWLEDGEMENT}

The authors would like to thank Pierre Michaud and Damien Fetis from IRISA/INRIA, France, for the interesting discussions and generous help on FreeFEM3d. We also thank Jeff Ng, Nozar Nozarian and Miles McGowan from ChipEstimate Inc. for their help with InCyte. This work is funded by NSF CRI grant CNS0551630 and has partial support from a MARCO IFC grant.

\section{REFERENCES}

[1] The International Technology Roadmap for Semiconductors (ITRS), 2003.

[2] W. Huang, M. R. Stan, and K. Skadron. Parameterized physical compact thermal modeling. IEEE Transactions on Components and Packaging Technologies, 28(4):615-622, December 2005.

[3] K. Skadron, K. Sankaranarayanan, S. Velusamy, D. Tarjan, M. R. Stan, and W. Huang. Temperature-aware microarchitecture: Modeling and implementation. ACM Transactions on Architecture and Code Optimization, 1(1):94-125, March 2004.

[4] W. Huang, K. Sankaranarayanan, R. J. Ribando, M. R. Stan, and K. Skadron. An improved HotSpot block-based thermal model with granularity considerations. In Workshop on Duplicating, Deconstructing, and Debunking (WDDD), in conjunction with Intl. Symp. on Computer Architecture (ISCA), June 2007.

[5] D. Brooks, V. Tiwari, and M. Martonosi. Wattch: A framework for architectural-level power analysis and optimizations. In Proc. Intl. Symp. of Computer Architecture (ISCA), pages 83-94, June 2000.

[6] W. Huang, M. R. Stan, K. Skadron, K. Sankaranarayanan, S. Ghosh, and S.Velusamy. Compact thermal modeling for temperature-aware design. In Proc. 41st Design Automation Conference (DAC), pages 878-883, June 2004.

[7] P. Chaparro, J. Gonzalez, G. Magklis, Q. Cai, and A. Gonzalez. Understanding the thermal implications of multicore architectures. IEEE Transactions on Parallel and Distributed Systems, 18(8):1055-65, 2007.

[8] Y. Yang, Z. P. Gu, C. Zhu, R. P. Dick, and L. Shang. ISAC: Integrated space and time adaptive chip-package thermal analysis. IEEE Transactions on Computer-Aided Design, 26(1):86-99, January 2007.

[9] W. Wu, L. Jin, J. Yang, P. Liu, and S. X.-D. Tan. Efficient power modeling and software thermal sensing for runtime temperature monitoring. ACM Transactions on Design Automation of Electronic Systems, 12(3), August 2007.

[10] W. Huang, S. Ghosh, S. Velusamy, K. Sankaranarayanan, K. Skadron, and M. R. Stan. HotSpot: A compact thermal modeling methodology for early-stage VLSI design. IEEE Transactions on Very Large Scale Integration (VLSI) Systems, 14(5):501-513, May 2006.

[11] N. Rinaldi. On the modeling of the transient thermal behavior of semiconductor devices. IEEE Transactions on Electronic Devices, 48(12):2796-1802, December 2001.

[12] D. Fetis and P. Michaud. An evaluation of HotSpot-3.0 block-based temperature model. In Workshop on Duplicating, Deconstructing, and Debunking (WDDD), in conjunction with Intl. Symp. on Computer Architecture (ISCA), June 2006.

[13] M. R. Stan, K. Skadron, M. Barcella, W. Huang, K. Sankaranarayanan, and S. Velusamy. HotSpot: a dynamic compact thermal model at the processor-architecture level. Microelectronics Journal, 34:1153-1165, 2003.

[14] T-Y. Wang and C. C-P. Chen. 3-D thermal-ADI: A linear-time chip level transient thermal simulator. IEEE Transactions on Computer-Aided Design of Integrated Circuits and Systems, 21(12):1434-1445, December 2002.

[15] H. Su, F. Liu, A. Devgan, E. Acar, and S. Nassif. Full chip estimation considering power supply and temperature variations. In Proc. Intl. Symp. of Low Power Elec. Design (ISLPED), pages 78-83, August 2003.

[16] P. Li, L. Pileggi, M. Asheghi, and R. Chandra. Efficient full-chip thermal modeling and analysis. In Proc. Intl. Conf. on Computer-Aided Design (ICCAD), 2004

[17] C. J. M. Lasance. Two benchmarks to facilitate the study of compact thermal modeling phenomena. IEEE Transactions on Components and Packaging Technologies, 24(4):559-565, December 2001.

[18] M-N. Sabry. Compact thermal models for electronic systems. IEEE Transactions on Components and Packaging Technologies, 26(1):179185, March 2003.

[19] E. G. T. Bosch. Thermal compact models: An alternative approach. IEEE Transactions on Components and Packaging Technologies, 26(1):173178, March 2003.

[20] W. Huang, E. Humenay, K. Skadron, and M. Stan. The need for a fullchip and package thermal model for thermally optimized IC designs. In Proc. Intl. Symp. on Low Power Electronic Design (ISLPED), pages 245-250, August 2005. 


\begin{tabular}{|c|c|c|c|c|c|}
\hline & active power & avg. temp rise & hottest temp rise & actual leakage & leakage error@ const temp \\
\hline $180 \mathrm{~nm}$, orig. design & $1.7 \mathrm{~W}$ & $30.77 \mathrm{C}$ & $59.98 \mathrm{C}$ & $546 \mu \mathrm{W}$ & $116 \%$ \\
\hline 90nm, scaled pow\&area & $316 \mathrm{~mW}$ & $6.19 \mathrm{C}$ & $25.42 \mathrm{C}$ & $24 \mathrm{~mW}$ & $66 \%$ \\
\hline 90nm, same pow\&area & $1.7 \mathrm{~W}$ & $>35.08 \mathrm{C}$ & runaway & $>277 \mathrm{~mW}$ & $>680 \%$ \\
\hline
\end{tabular}

TABLE I

AS TECHNOLOGY SCALES, TEMPERATURE DEPENDENCE OF SUBTHRESHOLD LEAKAGE POWER BECOMES MORE PROBLEMATIC. WITHOUT EARLY-STAGE THERMALLY OPTIMIZED DESIGN FLOW (FIG. 14), THERMAL RUNAWAY CAN HAPPEN EVEN FOR LOW-POWER SOC DESIGNS.

[21] K. Banerjee, S. C. Lin, A. Keshavarzi, and V. De. A self-consistent junction temperature estimation methodology for nanometer scale ICs with implications for performance and thermal management. In Proc. Intl. Elec. Dev. Meeting (IEDM), pages 36.7.1-36.7.4, 2003.

[22] L. He, W. Liao, and M. R. Stan. System level leakage reduction considering the interdependence of temperature and leakage. In Proc. 41st Design Automation Conference (DAC), pages 12-17, June 2004.

[23] P. Chaparro, J. Gonzalez, and A. Gonzalez. Thermal-effective clustered microarchitecture. In Proc. of First Workshop on Temperature-Aware Computer Systems, June 2004.

[24] E.C. Samson, S.V. Machiroutu, J.-Y. Chang, I. Santos, J. Hermerding, A. Dani, R. Prasher, and D.W.Song. Interface material selection and a thermal management technique in second-generation platforms built on Intel Centrino mobile technology. Intel Technology Journal, 9(1), February 2005.

[25] H. B. Bakoglu. Circuits, Interconnections, and Packaging for VLSI. Addison-Wesley Publishing Company, Reading, Massachusetts, 1990.

[26] Y. Zhang, D. Parikh, K. Sankaranarayanan, K. Skadron, and M. Stan. HotLeakage: A temperature-aware model of subthreshold and gate leakage for architects. Technical Report CS-2003-05, University of Virginia, Computer Science Department, 2003.

[27] H. Stolberg, S. Moch, L. Friebe, A. Dehnhardt, M. Kulaczewski, M. Berekovic, and P Pirsch. An SoC with two multimedia DSPs and a RISC core for video compression applications. In Digest of papers, IEEE International Solid-State Circuits Conference, February 2004.

[28] S. Heo, K. Barr, and K. Asanovic. Reducing power density through activity migration. In Proc. of Intl. Symp. on Low Power Electronics and Design (ISLPED'03), pages 217-222, August 2003.

[29] J. Srinivasan, S. V. Adve, P. Bose, and J. A. Rivers. The impact of technology scaling on lifetime reliability. In Proc. of The Intl. Conf. on Dependable Systems and Networks (DSN-04), June 2004.

[30] K. Sankaranarayanan, S. Velusamy, M.R. Stan, and K. Skadron. A case for thermal-aware floorplanning at the microarchitectural level. The Journal of Instruction-Level Parallelism, vol. 7, October 2005.

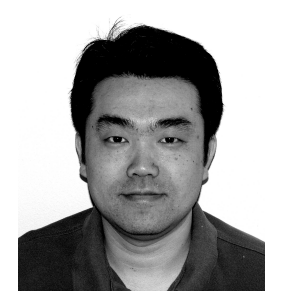

Wei Huang received a Ph.D. degree from the University of Virginia, and a B.E. degree from the University of Science and Technology of China, both in electrical engineering. He is currently with the Computer Science Department of University of Virginia as a postdoctoral researcher. His research interests include VLSI circuits and computer architecture with considerations of thermal, power, variability, and reliability issues.

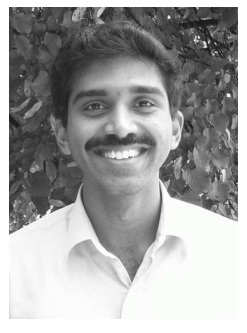

Karthik Sankaranarayanan received the B.E. degree in computer science and engineering from Anna University, Chennai, India, in 2000, and the M.S. degree from the University of Virginia, Charlottesville, in 2003. He is currently working toward the Ph.D. degree at the University of Virginia. He is a member of the LAVA Laboratory, University of Virginia, and his area of interests include computer architecture in general and thermal and power-aware microarchitectures in particular.

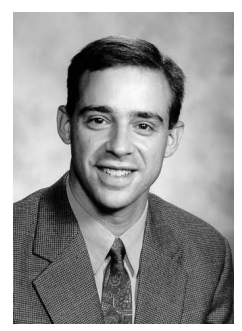

Kevin Skadron is an associate professor in the Department of Computer Science at the University of Virginia. Skadron's research interests focus on physical design challenges and programming models for multicore/manycore architectures, including graphics architectures. Skadron has a $\mathrm{PhD}$ in computer science from Princeton University and BS and BA degrees in Electrical and Computer Engineering and Economics from Rice University. $\mathrm{He}$ is cofounder and associate editor-in-chief of IEEE Computer Architecture Letters, a member of Eta Kappa $\mathrm{Nu}$, Omicron Delta Epsilon, and he is a senior member of the ACM, the IEEE, and the IEEE Computer Society and Circuits and Systems Society.

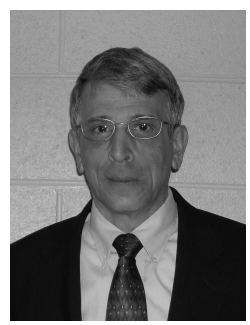

Robert J. Ribando is an associate professor in the Department of Mechanical and Aerospace Engineering at the University of Virginia. All his degrees are from Cornell University. Prior to coming to Virginia, he was on the research staff of the Advanced Reactors Safety Section at the Oak Ridge National Laboratory. His research and teaching interests include computational fluid dynamics and heat transfer and the graphical display of quantitative information. Applications have included nuclear reactor heat transfer, strongly rotating flows, biomedical flows, turbomachinery flows, etc. From 1992-1995 he held the Lucien Carr III Chair in Engineering Education, a temporary position intended to recognize and encourage the use of technology in instruction. He is currently writing a textbook and CD applying modern computational methods and visualization to the study of heat transfer.

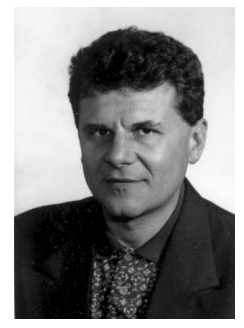

Mircea R. Stan received the Ph.D. (1996) and M.S. (1994) degrees in Electrical and Computer Engineering from the University of Massachusetts at Amherst and the Diploma (1984) in Electronics and Communications from "Politehnica" University in Bucharest, Romania. Since 1996 he has been with the Department of Electrical and Computer Engineering at the University of Virginia, where he is now an associate professor. Prof. Stan is teaching and doing research in the areas of high-performance low-power VLSI, temperature-aware circuits and architecture, embedded systems, and nanoelectronics. He has more than eight years of industrial experience, has been a visiting faculty at UC Berkeley in 2004-2005, at IBM in 2000, and at Intel in 2002 and 1999. He has received the NSF CAREER award in 1997 and was a co-author on best paper awards at GLSVLSI 2006, ISCA 2003 and SHAMAN 2002. He is the chair of the VLSI Systems and Applications Technical Committee (VSA-TC) of IEEE CAS, general chair for ISLPED 2006 and for GLSVLSI 2004, technical program chair for NanoNets 2007 and ISLPED 2005, and on technical committees for numerous conferences. He has been an Associate Editor for the IEEE Transactions on Circuits and Systems Systems I since 2004 and for the IEEE Transactions on VLSI Systems in 2001-2003. He has also been a Guest Editor for the IEEE Computer special issue on Power-Aware Computing in December 2003 and a Distinguished Lecturer for the IEEE Solid-State Circuits Society (SSCS) in 2007-2008, and for the IEEE Circuits and Systems (CAS) Society for 2004-2005. Prof. Stan is a senior member of the IEEE, a member of ACM, IET (former IEE), and also of Eta Kappa Nu, Phi Kappa Phi and Sigma Xi. 